\title{
SHCBP1 regulates apoptosis in lung cancer cells through phosphatase and tensin homolog
}

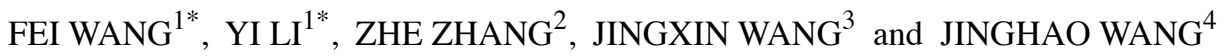 \\ ${ }^{1}$ Department of Thoracic Surgery, The Second Affiliated Hospital of Harbin Medical University; \\ ${ }^{2}$ Department of Neurology, Harbin Children's Hospital, Harbin, Heilongjiang 150086; \\ ${ }^{3}$ Department of Pharmacy, Hongqi Hospital of Mudanjiang Medical University, Mudanjiang, Heilongjiang 157011; \\ ${ }^{4}$ Department of Pharmacy, The First Affiliated Hospital, Jinan University, Guangzhou, Guangdong 510630, P.R. China
}

Received July 19, 2018; Accepted May 17, 2019

DOI: $10.3892 / \mathrm{ol} .2019 .10520$

\begin{abstract}
Src homologous and collagen (SHC) SH2-binding protein 1 (SHCBP1) is a member of the SHC family, and is overexpressed in numerous types of cancer. In addition, apoptosis serves an important role in the development of cancer. The purpose of this study was to examine the effect of SHCBP1 on apoptosis and its potential underlying mechanism in lung cancer cells. Apoptosis was detected by flow cytometry and caspase- 3 activity analysis. The expression levels of SHCBP1 and phosphatase and tensin homolog (PTEN) were detected by western blot analysis and reverse transcription-quantitative polymerase chain reaction. Cell viability was determined by MTT assay. The results indicated that SHCBP1 was increased in lung cancer cell lines and lung cancer tissues compared with in normal lung cell lines and tissues. The apoptosis of lung cancer cells was significantly increased by SHCBP1 small interfering RNA (siRNA), as indicated by the increased number of apoptotic cells and enhanced caspase- 3 activity. In addition, it was demonstrated that PTEN expression was modulated by SHCBP1 knockdown; silencing of SHCBP1 expression led to a significant increase in PTEN expression. Furthermore, inhibition of PTEN by siRNA reversed the increase in apoptosis induced by SHCBP1 siRNA. These results suggested that SHCBP1 may be upregulated in lung cancer and it may serve a key role in the apoptosis of lung cancer cells; this effect was associated with the expression of PTEN.
\end{abstract}

Correspondence to: Dr Jinghao Wang, Department of Pharmacy, The First Affiliated Hospital, Jinan University, 613 Huangpu Road, Tianhe, Guangzhou, Guangdong 510630, P.R. China

E-mail: wangjinghao_8585@163.com

*Contributed equally

Key words: lung cancer, SHC SH2-binding protein 1, phosphatase and tensin homolog, apoptosis

\section{Introduction}

Lung cancer is the leading cause of cancer-associated mortality, and non-small cell lung cancer (NSCLC) accounts for $>80 \%$ of lung cancer cases worldwide (1). Despite the fact that the diagnosis and treatment of lung cancer has improved, the 5-year survival rate of patients with NSCLC is <15\% (2). Understanding the molecular mechanisms underlying the effects of lung cancer-associated genes is important for identification of diagnostic and prognostic markers for the early detection and targeted treatment of lung cancer. Apoptosis serves an important role in the development and maintenance of multicellular organisms, and can remove damaged, aged or autoimmune cells (3). Apoptosis deficiency is one of the main characteristics of cancer cells. The primary goal of cancer treatment is to restore and activate apoptosis of cancer cells (4-6). However, to the best of our knowledge, an effective treatment that induces lung cancer cell apoptosis is not currently available. Therefore, identification of an optimal anti-lung cancer strategy that induces cell apoptosis is required.

Src homologous and collagen (SHC) is a cell-surface receptor adapter protein, which can activate growth factor receptors to signaling pathways, including insulin receptor, insulin growth factor receptor, epidermal growth factor receptor and fibroblast growth factor receptor (7-10). SHC SH2-binding protein 1 (SHCBP1) is a member of the SHC family, and has been recently identified in several malignant cancers (11). Aurora B-mediated SHCBP1 phosphorylation is essential for proper separation of the cleavage furrow (12). SHCBP1 has also been identified as a binding partner of central spindlin by proteome analysis (13). SHCBP1 activates the growth factor receptor, which has been indicated to serve a crucial role in proliferation and is disordered in a number of diseases, including cancer (14). SHCBP1 has also been reported to be overexpressed in human hepatocellular carcinoma (HCC) samples, whereas inhibition of SHCBP1 reduces cell proliferation and colony formation of HCC cells (15). In addition, SHCBP1 is increased in breast cancer, and serves a key role in cancer progression and apoptosis; therefore, it may be considered a potential predictor of breast cancer prognosis (11). However, the effect and underlying mechanism of SHCBP1 in lung cancer cell apoptosis remain unclear. The 
present study examined the role of SHCBP1 in lung cancer cell apoptosis.

Phosphatase and tensin homolog (PTEN) is a tumor suppressor gene on chromosome 10 that inhibits the proliferation, invasion and metastasis of tumor cells, and induces apoptosis of tumor cells, including lung cancer (16-18). The loss or mutation of the PTEN gene occurs in various human malignancies, and has been reported to be associated with increased tumor grade and adverse outcomes (16-18). Therefore, it was hypothesized that SHCBP1 may regulate PTEN in lung cancer cell apoptosis. The aim of the present study was to elucidate whether SHCBP1 inhibition induces apoptosis in lung cancer cells and to determine the relevant mechanisms.

\section{Materials and methods}

Patients and tissue samples. In the present study, NSCLC and normal tissue samples were obtained from 20 patients with NSCLC. A total of 10 patients were male and 10 patients were female; the median age at diagnosis was 63 years (range, 43-72 years). The samples were obtained from patients who were diagnosed with stage IA to IIIB NSCLC (19) and underwent surgery at the Second Affiliated Hospital of Harbin Medical University (Harbin, China) between July 2015 and September 2017. None of the patients received adjuvant chemotherapy, radiation or immunotherapy prior to surgery. For all patients who participated in this study, written informed consent was obtained, and this study was approved by the Ethical Committee of Harbin Medical University.

Immunohistochemistry. Tissue sections $(5 \mu \mathrm{m})$ were fixed with $10 \%$ formalin at room temperature for 20 min and embedded in paraffin, deparaffinized in xylene and rehydrated with graded alcohol. Endogenous peroxidase activity was blocked with $3 \%$ $\mathrm{H}_{2} \mathrm{O}_{2}$ for $15 \mathrm{~min}$ at room temperature, and tissue sections were cultured in a humidified atmosphere at $4^{\circ} \mathrm{C}$ with SHCBP1 mouse polyclonal antibodies (1:100; cat. no. ab122310; Abcam). The sections were then incubated at room temperature for $30 \mathrm{~min}$ with a secondary biotinylated antibody (1:500; cat. no. 14708; Cell Signaling Technology, Inc.). Antigen-antibody complexes were detected with the streptavidin-peroxidase $(15 \mathrm{~min})$ method with diaminobenzidine (DAB) as the chromogen substrate using the Vectastain elite ABC kit (Vector Laboratories, Inc.), according to the manufacturer's protocol. Peroxidase signals were observed visually following treatment with the DAB substrate-chromogen system for $8 \mathrm{~min}$; finally, the sections were stained with hematoxylin for $1 \mathrm{~min}$ at room temperature. PBS was used in place of the primary antibody as a negative control. Each slide was examined in five selected fields of view and 100 cells were observed using a Fluoview1000 laser scanning confocal microscope at x200 magnification (Olympus Corporation). Positive reactions were defined as those that indicated brown immune responses in the cytoplasm, nucleus and cell membrane.

Cell culture and transfection of SHCBP1 small interfering RNA (siRNA). The A549 human lung cancer cell line and the BEAS2B normal lung epithelial cell line were purchased from Shanghai Institute of Cell Biology, the Chinese Academy of Sciences. The cells were cultured in DMEM (Gibco; Thermo
Fisher Scientific, Inc.) supplemented with $10 \%$ fetal bovine serum (Gibco; Thermo Fisher Scientific, Inc.) at $37^{\circ} \mathrm{C}$ in an atmosphere containing $5 \% \mathrm{CO}_{2}$.

SHCBP1 siRNA was purchased from Abbexa Ltd. (cat. no. abx933286) and PTEN siRNA from Santa Cruz Biotechnology, Inc. (cat. no. sc-29459) Shanghai GenePharma Co., Ltd. synthesized the negative control (NC) siRNA. The siRNA-NC sequence was as follows: Forward, 5'-UUCUCC GAACGUGUCACGUTT-3' and reverse, 5'-ACGUGACAC GUUCGGAGAATT-3. According to the manufacturer's protocols, A549 cells were seeded in 6 -well plates $\left(1 \times 10^{5}\right.$ cells/well $)$ and starved in serum-free medium for $24 \mathrm{~h}$ prior to transfection for $72 \mathrm{~h}$ at room temperature using X-treme GENE siRNA transfection reagent (Roche Applied Science, Penzberg, Germany). The final concentration of SHCBP1 siRNA and siRNA-NC was $100 \mathrm{nM}$, and of PTEN siRNA was $200 \mathrm{nM}$.

Cell viability assay. The viability of A549 cells was determined by MTT assay (Sigma-Aldrich; Merck KGaA), according to the manufacturer's protocol. Briefly, cells $\left(2 \times 10^{4}\right.$ cells/well $)$ were seeded in a 96-well culture plate. Cells were transfected with SHCBP1 siRNA or PTEN siRNA for $72 \mathrm{~h}$. Following incubation, $5 \mathrm{mg} / \mathrm{ml}$ MTT solution was added and cells were incubated for a further $4 \mathrm{~h}$ at $37^{\circ} \mathrm{C}$. MTT formazan crystals were solubilized with DMSO and absorbance was measured at a wavelength of $490 \mathrm{~nm}$ using a microplate reader. Cell viability was measured as percentage of viable cells relative to the control cells.

Flow cytometry. Apoptotic A549 cells were analyzed using the Alexa Fluor ${ }^{\circledR} 488$ Annexin V/Dead Cell Apoptosis kit (Invitrogen; Thermo Fisher Scientific, Inc.), according to the manufacturer's protocol. A549 cells were transfected with SHCBP1 siRNA for $72 \mathrm{~h}$, and were then collected and washed with cold PBS. Following centrifugation at $450 \mathrm{x}$ g for $4 \mathrm{~min}$ at room temperature, the supernatant was discarded and cell pellets were re-suspended in $1 \mathrm{X}$ Annexin-binding buffer to a final concentration of $1 \times 10^{6}$ cells $/ \mathrm{ml}$. Subsequently, $1 \mu \mathrm{l}$ Alexa Fluor $^{\circledR} 488$ Annexin V and $1 \mu 1100 \mu \mathrm{g} / \mathrm{ml}$ propidium iodide working solution were added to each $100 \mu \mathrm{l}$ cell suspension. Cells were incubated in the dark at room temperature for $15 \mathrm{~min}$. Stained cells were detected by fluorescence spectrometry at 530 and $575 \mathrm{~nm}$. The apoptotic rate was calculated by BD FACSDiva software (BD Biosciences).

Reverse transcription-quantitative polymerase chain reaction (PCR). Total RNA from lung tissues, A549 and BEAS2B cells was extracted with TRIzol ${ }^{\circledR}$ reagent (Invitrogen; Thermo Fisher Scientific, Inc.), according to the manufacturer's protocol. The SuperScript IV Reverse Transcriptase kit (Thermo Fisher Scientific, Inc.) was used to synthesize cDNA according to manufacturer's protocol. The mRNA expression levels of SHCBP1 and PTEN were assessed by SYBR Green incorporation on a Roche LightCycler ${ }^{\circledR} 480$ real-time PCR system (Roche Diagnostics, Indianapolis, IN, USA), with GAPDH as an internal control. The thermocycling conditions were as follows: $120 \mathrm{sec}$ at $95^{\circ} \mathrm{C}$, followed by 40 cycles at $95^{\circ} \mathrm{C}$ for $30 \mathrm{sec}$ and $55^{\circ} \mathrm{C}$ for $40 \mathrm{sec}$, and a final step extension at $72^{\circ} \mathrm{C}$ for $3 \mathrm{~min}$. Data was collected at the end of the extension step $\left(72^{\circ} \mathrm{C}\right)$. The primer sequences were: SHCBP1, forward 
5'-GCTACCGTGATAAACCAGGTTC-3', reverse 5'-AGG CTCTGAATCGCTCATAGA-3'; PTEN, forward 5'-TGGATT CGACTTAGACTTGACCT-3', reverse 5'-GCGGTGTCATAA TGTCTCTCAG-3'; and GAPDH, forward 5'-AAGAAGGTG GTGAAGCAGGC-3' and reverse, 5'-TCCACCACCCAGTTG CTGTA-3'. The expression levels of the target genes were obtained by normalizing to the endogenous reference gene and were compared with a calibrator (average of the control samples), using the $2^{-\Delta \Delta \mathrm{Cq}}$ method (20).

Western blot analysis. Protein samples from lung tissues, A549 and BEAS2B cells were analyzed (21). Protein samples $(70 \mu \mathrm{g})$ were extracted with lysis buffer (Beyotime Institute of Biotechnology) supplemented with $1 \%$ protease inhibitor solution. The concentration of the proteins was determined using a BCA Protein Assay kit (Beyotime Institute of Biotechnology). Subsequently, proteins $(20 \mu \mathrm{g})$ were separated by $10 \%$ SDS-PAGE and transferred to nitrocellulose membranes. The membranes were blocked in PBS containing 5\% non-fat milk for $2 \mathrm{~h}$ at room temperature and incubated at $4^{\circ} \mathrm{C}$ overnight with the following primary antibodies: SHCBP1 $(1: 1,000$; cat. no. ab184467; Abcam), PTEN (1:500; cat. no. ab32199; Abcam) and GAPDH (1:2,000; cat. no. TA802519; OriGene Technologies, Inc.). Following washing, the membrane was incubated with secondary anti-rabbit immunoglobulin $\mathrm{G}$ (IgG; 1:1,000; cat. no. A3687; Sigma-Aldrich; Merck KGaA) and anti-mouse $\operatorname{IgG}$ (1:1,000; cat. no. M8770; Sigma-Aldrich; Merck KGaA) antibodies at room temperature for $1 \mathrm{~h}$. Images were captured on an Odyssey CLx Infrared Imaging system (LI-COR Biosciences). Subsequently, the blots were semi-quantified using Odyssey CLx 2.1 software (LI-COR Biosciences). Data were normalized to GAPDH as an internal control.

Caspase-3 activity assay. According to the manufacturer's protocol, caspase-3 activity in A549 cells was determined using a colorimetric assay kit (cat. no. C1116; Beyotime Institute of Biotechnology). A549 cell lysis was performed in ice for $15 \mathrm{~min}$, and the lysate was subsequently centrifuged at $20,000 \mathrm{xg}$ for $10 \mathrm{~min}$ at $4^{\circ} \mathrm{C}$. Supernatant samples $(30 \mu \mathrm{l})$ were then incubated with $10 \mu \mathrm{l}$ substrate ( $2 \mathrm{mM}$ Ac-DEVD-pNA) in $60 \mu \mathrm{l}$ assay buffer at $37^{\circ} \mathrm{C}$ for $2 \mathrm{~h}$. The absorbance rate was measured at a wavelength of $405 \mathrm{~nm}$.

Statistical analysis. Group data are expressed as the mean \pm standard error of the mean and were analyzed by SPSS 17.0 software (SPSS, Inc.). Student's t-test was performed for two-group comparisons. One-way analysis of variance followed by Dunnett's test was used for multiple-group comparisons. $\mathrm{P}<0.05$ was considered to indicate a statistically significant difference. Figures were constructed using GraphPad Prism 5.0 software (GraphPad Software, Inc.).

\section{Results}

SHCBP1 expression is upregulated in lung cancer tissues. To detect SHCBP1 expression in lung cancer, the expression of SHCBP1 was compared in 20 lung cancer tissues and adjacent normal tissues. Human normal lung tissues did not exhibit SHCBP1 protein expression by immunostaining, whereas the proportion of SHCBP1-positive cells in lung cancer samples was higher compared with in the normal lung tissue samples (Fig. 1A and B). SHCBP1 was revealed to be located in the cytoplasm. These results indicated that SHCBP1 was overexpressed in lung cancer cells. As shown in Fig. 1C and D, the mRNA and protein expression levels of SHCBP1 were significantly higher in lung cancer tissues compared with in adjacent normal tissues. These results suggested that SHCBP1 may be significantly overexpressed in lung cancer and may serve an important role in the tumorigenesis of lung cancer.

Stable SHCBP1 siRNA transfection in lung cancer cell lines. SHCBP1 was measured in A549 cells and the normal lung cell line, BEAS2B (Fig. 2A and B). The results indicated that compared with in BEAS2B cells, SHCBP1 was highly expressed in the tumor cell line A549. The efficiency of SHCBP1 knockdown by siRNA was verified by detection of mRNA and protein expression levels, which were significantly reduced compared with in A549 cells transfected with siRNA-NC at $24 \mathrm{~h}$ (Fig. 2C and D). The results indicated that siRNA can be used to generate stable SHCBP1 knockdown in A549 cells.

Inhibition of SHCBP1 promotes apoptosis of lung cancer cells. To examine the possible role and underlying mechanism of SHCBP1 in lung cancer cells, siRNA was used to suppress SHCBP1 expression, and caspase-3 and cell apoptosis activity were determined. As shown in Fig. 3A, the viability of A549 cells was reduced by SHCBP1 siRNA transfection at 24,48 and $72 \mathrm{~h}$; the most obvious decrease in cell viability was detected at $72 \mathrm{~h}$. Therefore, in A549 cells, SHCBP1 siRNA transfection at $72 \mathrm{~h}$ was used for subsequent experiments. SHCBP1 siRNA-induced apoptosis was further confirmed by flow cytometry, and an increased number of apoptotic A549 cells was detected at $72 \mathrm{~h}$ following SHCBP1 siRNA transfection (Fig. 3B and C). Furthermore, alterations in caspase- 3 activity, a key downstream protease that executes the lung cancer apoptotic cascade, were measured; caspase-3 activity is considered to be the last step of caspase-dependent apoptosis $(6,21,22)$. As shown in Fig. 3D, SHCBP1 siRNA increased the levels of caspase-3 activity compared with siRNA-NC in A549 cells. Based on these findings, it was concluded that SHCBP1 siRNA may induce apoptotic effects on lung cancer cells.

SHCBPI siRNA promotes lung cancer cell apoptosis partly via PTEN. PTEN serves an important role in apoptosis of various types of cancer cells, including lung cancer $(17,23)$. Therefore, the present study investigated whether PTEN was involved in SHCBP1-mediated apoptosis. The efficiency of PTEN knockdown was validated by detection of mRNA and protein expression levels, which were lower in A549 cells compared with in cells transfected with siRNA-NC (Fig. 4A and B). Subsequently the effects of SHCBP1 siRNA on PTEN expression were assessed in A549 cells. As shown in Fig. 4C and D, SHCBP1 siRNA significantly upregulated the expression levels of PTEN in A549 cells. Following transfection of A549 cells with SHCBP1 siRNA and PTEN siRNA, PTEN expression was significantly decreased compared with in cells transfected with SHCBP1 siRNA alone. The role of PTEN siRNA in SHCBP1 siRNA-induced apoptosis was 


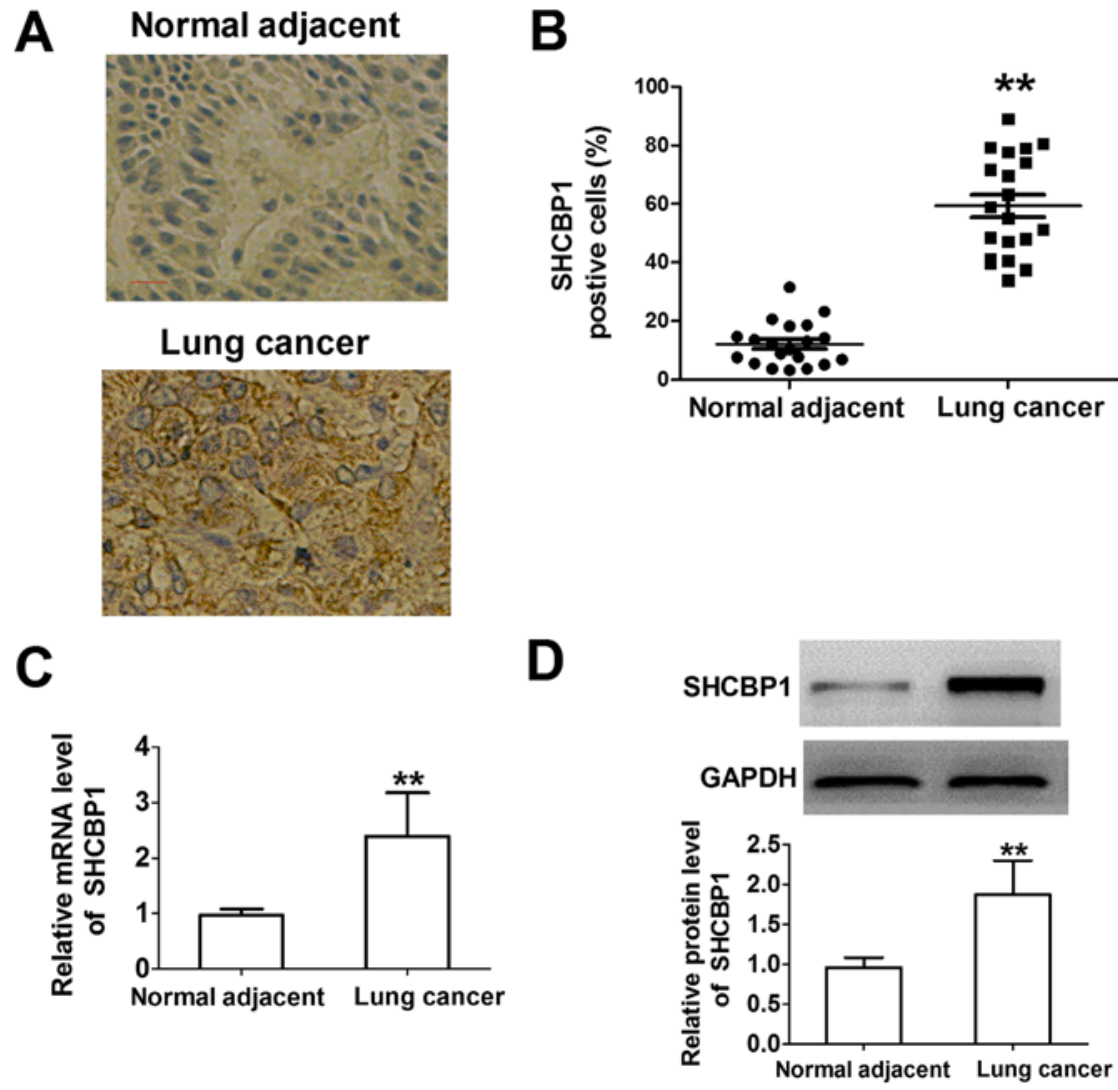

Figure 1. Expression of SHCBP1 is upregulated in lung cancer tissues. (A) Representative images of SHCBP1 immunohistochemical staining. Magnification, $\mathrm{x} 200$; scale bar, $50 \mathrm{~mm}$. (B) Number of SHCBP1-positive cells. ${ }^{* *} \mathrm{P}<0.01$ vs. normal adjacent group, $\mathrm{n}=20$ /group. (C) Relative expression levels of SHCBP1 in lung cancer tissues and normal adjacent tissues. N=6 samples/group. (D) Representative western blot images of SHCBP1 protein and semi-quantification of results indicating SHCBP1 protein expression levels. N=4 samples/group. ${ }^{* *} \mathrm{P}<0.01$ vs. normal adjacent group. SHCBP1, SHC SH2-binding protein 1.

A

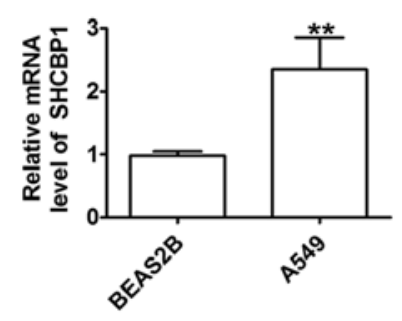

B

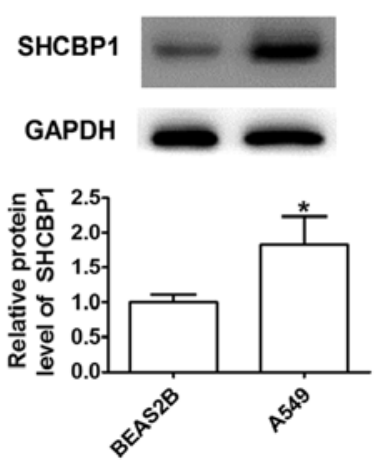

C

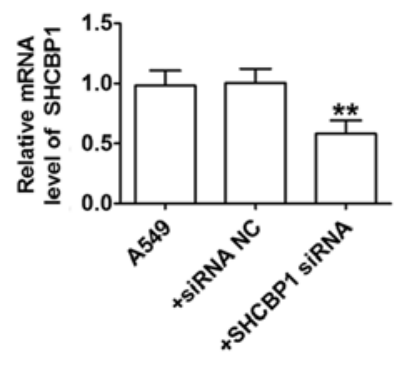

D SHCBP1

GAPDH

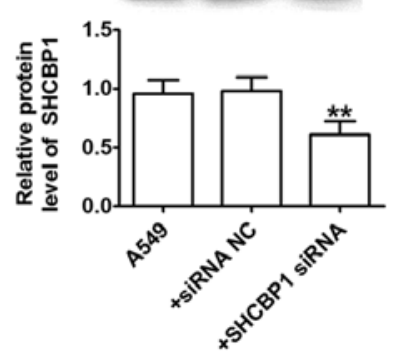

Figure 2. Stable SHCBP1 siRNA transfection in lung cancer cell lines. (A and C) mRNA expression levels of SHCBP1. (B and D) Representative western blot images of SHCBP1 protein. (A and B) ${ }^{*} \mathrm{P}<0.05$ and ${ }^{* *} \mathrm{P}<0.01$ vs. BEAS2B cells. (C and D) ${ }^{* *} \mathrm{P}<0.01$ vs. A549 and siRNA NC groups. N=4. SHCBP1, SHC SH2-binding protein 1; siRNA, small interfering RNA.

further tested. As indicated in Fig. 4E-G, SHCBP1 siRNA increased the number of apoptotic cells, reduced cell viability and increased caspase-3 activity in A549 cells; these effects that were reversed following PTEN-knockdown with siRNA. Therefore, SHCBP1 siRNA may promote the apoptosis of lung cancer cells at least in part through PTEN induction.

\section{Discussion}

The present study indicated that SHCBP1 was overexpressed in lung cancer cell lines and lung cancer tissues. siRNA-induced knockdown of SHCBP1 significantly increased the apoptosis of lung cancer cells. In addition, SHCBP1 siRNA significantly increased the expression of PTEN, whereas inhibition of PTEN reversed SHCBP1 siRNA-induced apoptosis. These results suggested that SHCBP1 may be upregulated in lung cancer and may serve an important role in the apoptosis of lung cancer cells, which may be associated with PTEN. The present study elucidated a potential novel effect and mechanism of SHCBP1 on apoptosis of lung cancer cells.

Lung cancer is one of the most common malignancies and the leading cause of cancer-associated mortality. It is a complex 

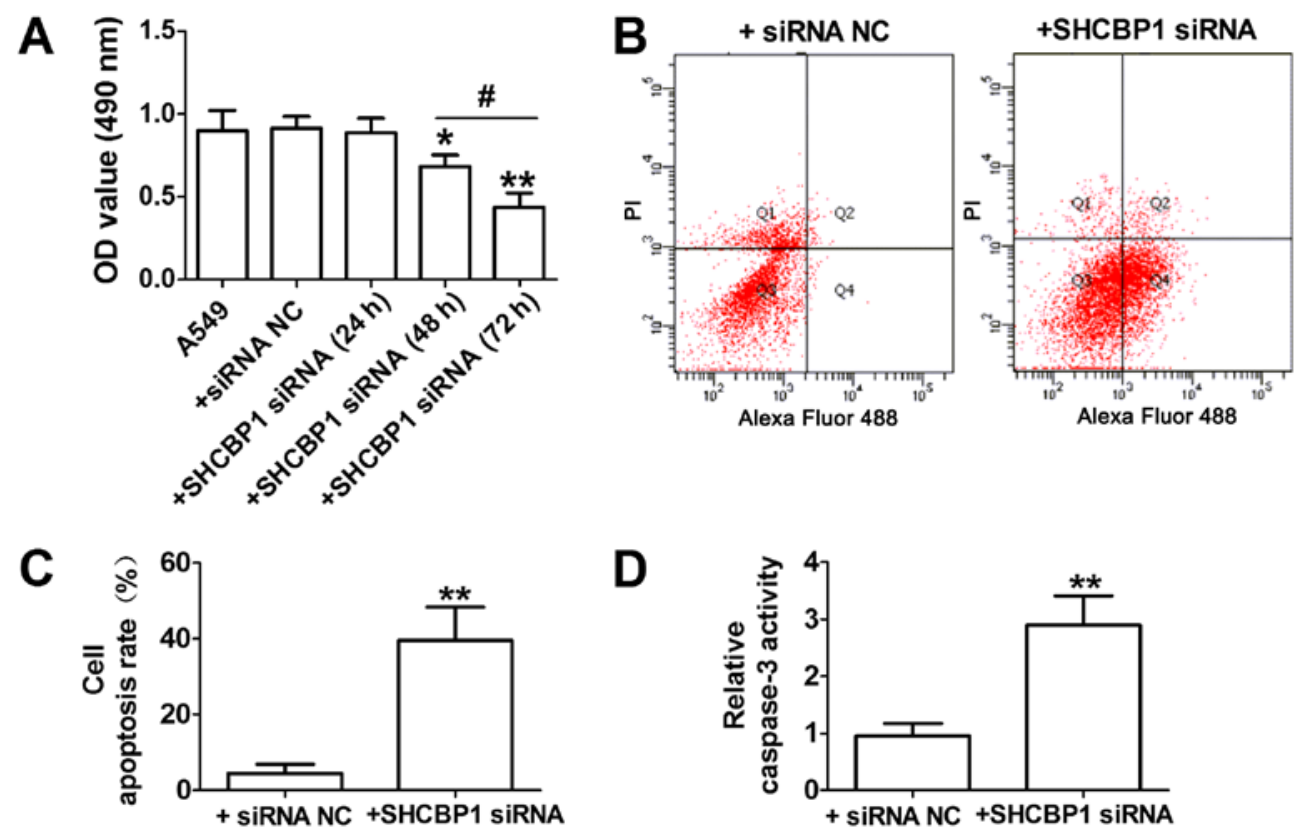

Figure 3. Inhibition of SHCBP1 expression promotes apoptosis of lung cancer cells. (A) MTT assay results. "P<0.05 and ${ }^{* *} \mathrm{P}<0.01$ vs. A549 and siRNA NC groups; " $\mathrm{P}<0.05$ SHCBP1 siRNA (48 h) vs. SHCBP1 siRNA (72 h). (B) Representative images of flow cytometry produced by Annexin V and PI staining. Right upper quadrant: Cells in late apoptosis. Right lower quadrant: Cells in early apoptosis. Left lower quadrant: Viable cells. Left upper quadrant: Necrotic cells. (C) Proportion of early and late apoptotic cells in A549 cells. (D) Caspase-3 activity. ${ }^{* *} \mathrm{P}<0.01$ vs. siRNA NC group. N=4. FITC, fluorescein isothiocyanate; OD, optical density; PI, propidium iodide; SHCBP1, SHC SH2-binding protein 1; siRNA, small interfering RNA.

A

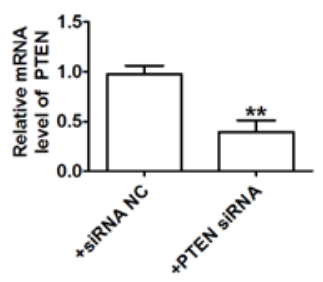

B

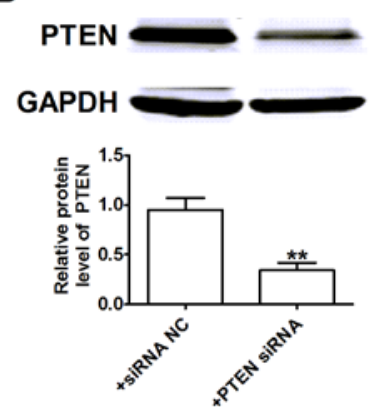

C

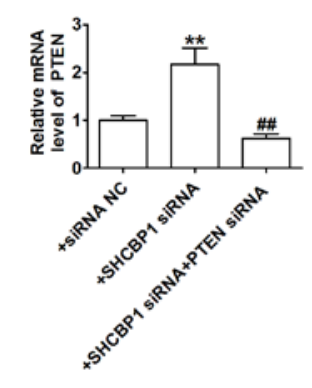

E
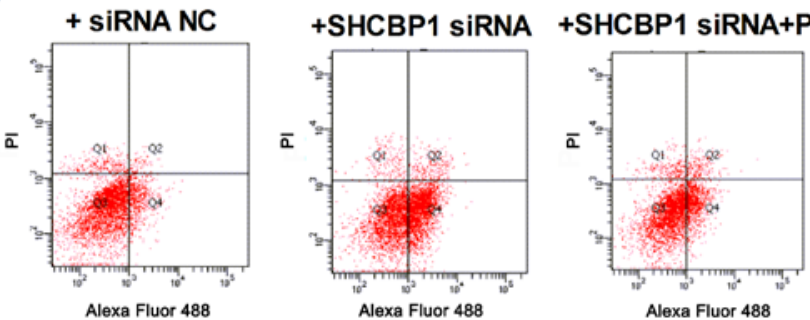

$\mathbf{F}$
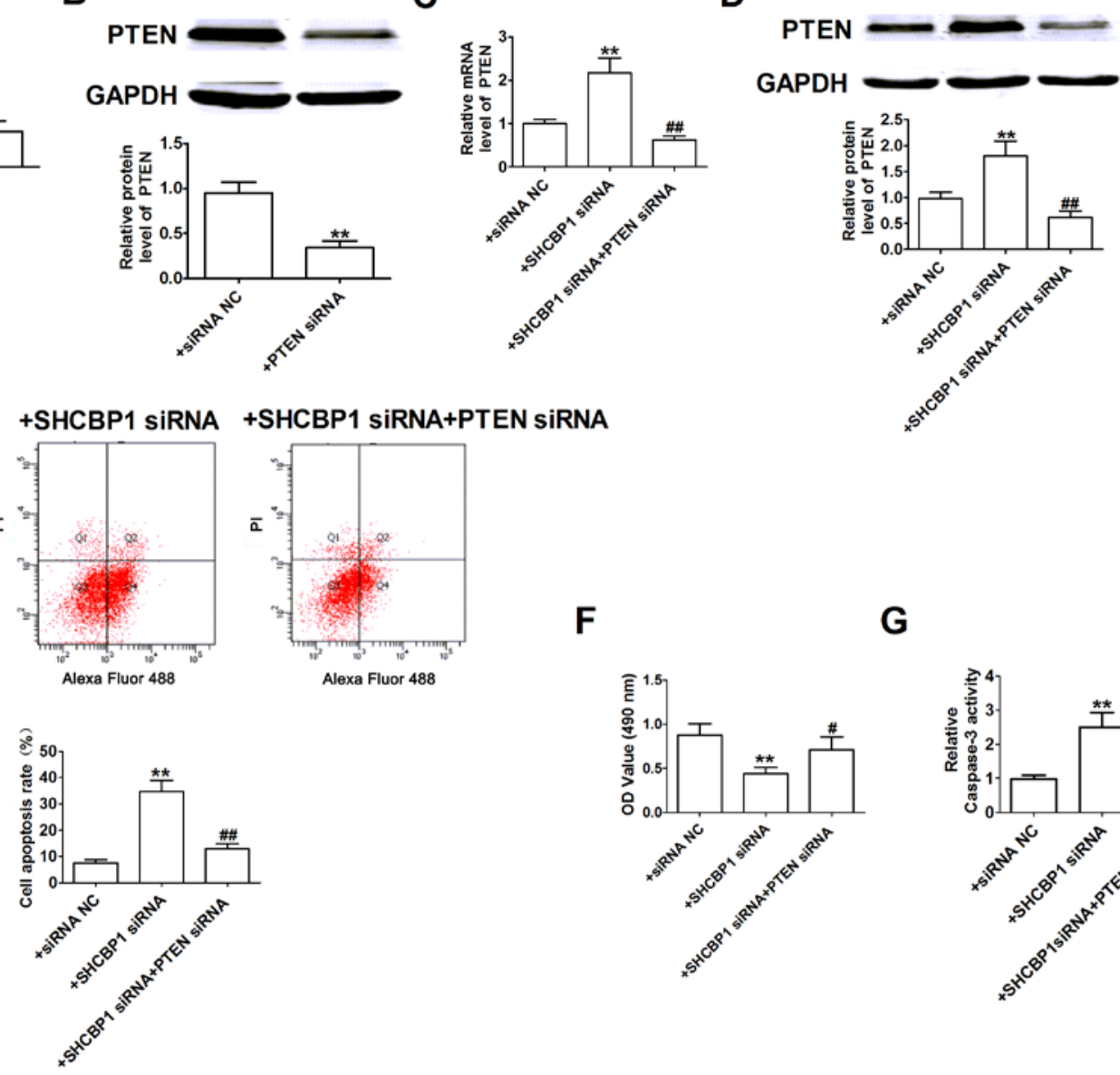

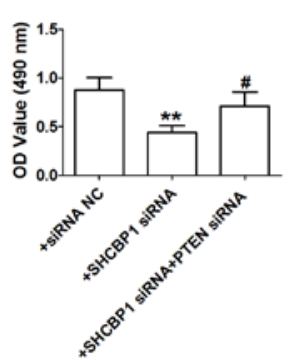

G

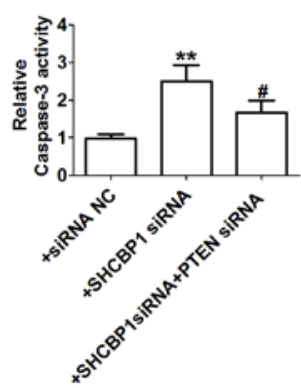

Figure 4. SHCBP1 siRNA promotes lung cancer cell apoptosis partly via PTEN. (A and C) mRNA expression levels of PTEN. (B and D) Representative western blot images of PTEN protein. (E) Representative images of flow cytometry and proportion of early and late apoptotic cells in A549 cells. (F) MTT assay results. (G) Caspase-3 activity. ${ }^{* *} \mathrm{P}<0.01$ vs. siRNA NC; ${ }^{~} \mathrm{P}<0.05$ and ${ }^{\# \#} \mathrm{P}<0.01$ vs. SHCBP1 siRNA. N=4. OD, optical density; PI, propidium iodide; $\mathrm{PTEN}$, phosphatase and tensin homolog; SHCBP1, SHC SH2-binding protein 1; siRNA, small interfering RNA. 
disease associated with numerous dysregulated signaling pathways, including apoptosis $(1,3,4)$. Numerous natural and synthetic compounds have been reported to exert anticancer effects by inducing various apoptotic pathways $(6,24,25)$. A better understanding of the function of lung cancer cell apoptosis may provide novel therapeutic approaches for disease prevention or control.

SHCBP1 is a member of the SHC family. SHC genes have been reported to be important in the regulation of mammalian apoptosis and resistance (11). Dysregulated SHCs may lead to uncontrolled proliferation, which is a hallmark of human cancer (11). SHCBP1 is overexpressed in HCC, and the loss of SHCBP1 in HCC cells leads to inhibition of cell proliferation and increased apoptosis (15). In addition, SHCBP1 is associated with the spread and apoptosis of breast cancer (11). However, to the best of our knowledge, the expression of SHCBP1, and the possible role and mechanisms of SHCBP1 in the apoptosis of lung cancer cells have not been studied. The present study hypothesized that SHCBP1 may be associated with lung cancer.

To confirm the role of SHCBP1 in lung cancer cell apoptosis, the expression levels of SHCBP1 were examined in lung cancer and matched normal lung tissue samples. The results indicated a significant increase in SHCBP1 expression in cancer compared with in normal lung tissue, suggesting that SHCBP1 may be a candidate oncogene in lung cancer. SHCBP1 siRNA was subsequently transfected into the lung cancer cell line A549 to inhibit its expression. The in vitro experiments indicated that inhibition of SHCBP1 significantly increased apoptosis of lung cancer cells and caspase-3 activity, and inhibited cell viability. These results suggested that SHCBP1 siRNA may promote the apoptosis of lung cancer cells.

PTEN is a well-known tumor suppressor gene, which has been indicated to serve a crucial role in the spread, apoptosis and invasion of lung cancer $(16,17)$. Therefore, the association between SHCBP1 and PTEN was examined in lung cancer cells. The results indicated that PTEN expression was significantly increased in SHCBP1-knockdown A549 cells. In addition, PTEN siRNA reversed the effects of SHCBP1 siRNA on apoptosis, caspase-3 activity and cell viability. All these results indicated that SHCBP1 siRNA may promote apoptosis of lung cancer cells partly by upregulating PTEN. However, the involvement of other mechanisms that may mediate the pro-apoptotic effects of SHCBP1 siRNA cannot be excluded. Future research may focus on disrupting the interactions between SHCBP1 and downstream targets, which may have important therapeutic implications. Our subsequent studies will focus on collecting more samples to examine pathological and clinical data of patients with NSCLC in order to analyze SHCBP1 further, and will investigate whether it is significantly associated with invasion depth, lymph node metastasis, tumor size and survival.

In conclusion, the present study indicated that SHCBP1 may serve an important role in regulating apoptosis of lung cancer cells. In addition, it was suggested that this role may be regulated by PTEN. Therefore, the candidate oncogene SHCBP1 may be considered an effective novel therapeutic target for the treatment of lung cancer.

\section{Acknowledgements}

Not applicable.

\section{Funding}

The present study was supported in part by 2018 Jinan University First Clinical Medical College Research and Cultivation Fund Project (grant no. 2018104 to JHW).

\section{Availability of data and materials}

The datasets used and analyzed during the present study are available from the corresponding author upon reasonable request.

\section{Authors' contributions}

FW and JHW were responsible for the conception and design of the study. FW, YL, ZZ and JXW performed the experiments. FW analyzed and interpreted the data. FW and JHW drafted the article and were responsible for the revision of the manuscript. JHW gave final approval of the version to be published.

\section{Ethics approval and consent to participate}

For all patients who participated in this study, written informed consent was obtained, and this study was approved by the Ethical Committee of Harbin Medical University.

\section{Patient consent for publication}

Not applicable.

\section{Competing interests}

The authors declare that they have no competing interests.

\section{References}

1. Nana-Sinkam SP and Powell CA: Molecular biology of lung cancer: Diagnosis and management of lung cancer, 3rd edition: American college of chest physicians evidence-based clinical practice guidelines. Chest 143: e30S-e39S, 2013.

2. Reungwetwattana T and Dy GK: Targeted therapies in development for non-small cell lung cancer. J Carcinog 12: 22, 2013.

3. Othman $\mathrm{N}$ and Nagoor NH: The role of microRNAs in the regulation of apoptosis in lung cancer and its application in cancer treatment. Biomed Res Int 2014: 318030, 2014.

4. Hassan M, Watari H, AbuAlmaaty A, Ohba Y and Sakuragi N: Apoptosis and molecular targeting therapy in cancer. Biomed Res Int 2014: 150845, 2014.

5. Tsuruo T, Naito M, Tomida A, Fujita N, Mashima T, Sakamoto H and Haga N: Molecular targeting therapy of cancer: Drug resistance, apoptosis and survival signal. Cancer Sci 94: 15-21, 2003.

6. Ding Y and Nguyen TA: Pq1, a quinoline derivative, induces apoptosis in T47D breast cancer cells through activation of caspase-8 and caspase-9. Apoptosis 18: 1071-1082, 2013.

7. Ornitz DM and Itoh N: The fibroblast growth factor signaling pathway. Wiley Interdiscip Rev Dev Biol 4: 215-266, 2015.

8. Koul HK, Pal M and Koul S: Role of p38 MAP kinase signal transduction in solid tumors. Genes Cancer 4: 342-359, 2013

9. Chen Y, Long H, Wu Z, Jiang X and Ma L: EGF transregulates opioid receptors through EGFR-mediated GRK2 phosphorylation and activation. Mol Biol Cell 19: 2973-2983, 2008.

10. Qu WS, Tian DS, Guo ZB, Fang J, Zhang Q, Yu ZY, Xie MJ, Zhang HQ, Lu JG and Wang W: Inhibition of EGFR/MAPK signaling reduces microglial inflammatory response and the associated secondary damage in rats after spinal cord injury. J Neuroinflammation 9: 178, 2012. 
11. Feng W, Li HC, Xu K, Chen YF, Pan LY, Mei Y, Cai H, Jiang YM, Chen T and Feng DX: SHCBP1 is over-expressed in breast cancer and is important in the proliferation and apoptosis of the human malignant breast cancer cell line. Gene 587: 91-97, 2016.

12. Asano E, Hasegawa H, Hyodo T, Ito S, Maeda M, Chen D, Takahashi M, Hamaguchi M and Senga T: SHCBP1 is required for midbody organization and cytokinesis completion. Cell Cycle 13: 2744-2751, 2014

13. Buckley MW, Arandjelovic S, Trampont PC, Kim TS, Braciale TJ and Ravichandran KS: Unexpected phenotype of mice lacking SHCBP1, a protein induced during T cell proliferation. PLoS One 9: e105576, 2014

14. Kupershmidt I, Su QJ, Grewal A, Sundaresh S, Halperin I, Flynn J, Shekar M, Wang H, Park J, Cui W, et al: Ontology-based meta-analysis of global collections of high-throughput public data. PLoS One 5: pii: e13066, 2010.

15. Tao HC, Wang HX, Dai M, Gu CY, Wang Q, Han ZG and Cai B: Targeting SHCBP1 inhibits cell proliferation in human hepatocellular carcinoma cells. Asian Pac J Cancer Prev 14: 5645-5650, 2013.

16. Carracedo A, Alimonti A and Pandolfi PP: PTEN level in tumor suppression: How much is too little? Cancer Res 71: 629-633, 2011.

17. Hollander MC, Blumenthal GM and Dennis PA: PTEN loss in the continuum of common cancers, rare syndromes and mouse models. Nat Rev Cancer 11: 289-301, 2011.

18. Lu XX, Cao LY, Chen X, Xiao J,Zou Y and Chen Q: PTEN inhibits cell proliferation, promotes cell apoptosis and induces cell cycle arrest via downregulating the PI3K/AKT/hTERT pathway in lung adenocarcinoma A549 cells. Biomed Res Int 2016: 2476842, 2016.

19. Lu S, Xu J, Xu X, Hu S, Li B and Li W: The expression of astrocyte elevated gene-1 in human non-small-cell lung cancer and its relationship with postoperative chemotherapy and radiotherapy. Histopathology 67: 817-826, 2015.
20. Livak KJ and Schmittgen TD: Analysis of relative gene expression data using real-time quantitative PCR and the 2(-Delta Delta C(T)) method. Methods 25: 402-408, 2001.

21. Chu C, Liu X, Bai X, Zhao T, Wang M, Xu R, Li M, Hu Y, Li W, Yang L, et al: MiR-519d suppresses breast cancer tumorigenesis and metastasis via targeting MMP3. Int J Biol Sci 14: 228-236, 2018.

22. Xue Y, Wu L, Liu Y, Ma Y, Zhang L, Ma X, Yang Y and Chen J: ENTPD5 induces apoptosis in lung cancer cells via regulating caspase 3 expression. PLoS One 10: e0120046, 2015.

23. Guo Y, Chang H, Li J, Xu XY, Shen L, Yu ZB and Liu WC: Thymosin alpha 1 suppresses proliferation and induces apoptosis in breast cancer cells through PTEN-mediated inhibition of PI3K/Akt/mTOR signaling pathway. Apoptosis 20: 1109-1121, 2015.

24. Shimizu S: development of anti-cancer drugs mediated by apoptosis and autophagy. Nihon Rinsho 73: 1302-1307, 2015 (In Japanese)

25. Gali-Muhtasib H, Hmadi R, Kareh M, Tohme R and Darwiche N: Cell death mechanisms of plant-derived anticancer drugs: Beyond apoptosis. Apoptosis 20: 1531-1562, 2015.

(i) $($ ) This work is licensed under a Creative Commons Attribution-NonCommercial-NoDerivatives 4.0 International (CC BY-NC-ND 4.0) License. 